\title{
A Method for Spatial Signature Estimation with Uncalibrated Uniform Linear Array
}

\author{
Xiang Cao ${ }^{\# * 1}$, Jingmin Xin ${ }^{*}$, Yoshifumi Nishio ${ }^{* 2}$, Nanning Zheng *, Akira Sano ${ }^{\dagger 3}$ \\ \# Institute of Artificial Intelligence and Robotics, Xi'an Jiaotong University \\ $X i$ 'an 710049, China \\ ${ }^{1}$ cao.xiangestu.xjtu.edu.cn \\ * Department of Electrical and Electronic Engineering, The University of Tokushima \\ Tokushima 770-8506, Japan \\ 2 nishiodee.tokushima-u.ac.jp \\ ${ }^{\dagger}$ Department of System Design Engineering, Keio University \\ Yokohama 223-8522, Japan \\ ${ }^{3}$ sanolsd.keio.ac.jp
}

\begin{abstract}
In this paper, a multidimensional search procedure is presented for estimating spatial signature with uniform linear array in the presence of unknown gain and phase errors. The proposed method fully exploits multiple invariances in the interior of the sensor array and it is desirable that the quality of estimate improves as more and more array invariances are used. A Gauss-Newton iterative algorithm is applied for solving the multidimensional nonlinear problem. Simulation results indicate that a solution close to the minimum is obtained after only two or three iterations. Furthermore, the introduction of a weighting matrix, similar to weighted subspace fitting, can tone up the performance of our method.
\end{abstract}

\section{INTRODUCTION}

In sensor array signal processing, many high-resolution methods such as ESPRIT [1] have been advanced for estimation of unknown parameters embedded in array output model. It is a natural motivation of array calibration, since the performance of these methods will degrade dramatically when the ideal array model is damaged by some unknown errors (e.g., mutual coupling and sensor position uncertainties).

Two parametric errors (i.e., statistical and deterministic) were usually used to extend the ideal array model. This paper only focus on the deterministic unknown gain and phase errors. The term deterministic here implies that the unknown error at each of elements of array is a complex stable constant during the period of observation.

Several so-called auto-calibration approaches have been developed in literature. Here, the auto-calibration indicates that array calibration may be accomplished without employing any dummy elements or transmitters at known direction. In [2], an iterative eigenstructure-based technique of estimating Directions-of-Arrival (DOA's) in the presence of unknown gain and phase errors is presented and it can apply to arbitrary array geometries except uniform linear array (ULA). For ULA, a phase ambiguity exists between the diagonal error matrix and the ideal array steering matrix (see [3] and [4]). Thus, it is impossible to estimate DOA and gain and phase errors simultaneously for ULA. A Hermitian Toeplitz structure of
ULA output covariance matrix in the absence of gain and phase errors is exploited in [5] and [6]. This method takes advantage of the elements equivalence at every diagonal line to form two equations for estimation of gain and phase errors. The main drawback of the approach is that the Toeplitz matrix assumption is only established under infinite sampling condition. It means that if the number of snapshots is fixed, the performance of the algorithm does not improve after increasing the signal-to-noise ratio (SNR) to a certain point. In addition, ESPRIT method also can be extended to the case. The idea firstly emerged from [7], and is applied for estimating spatial signature in [8] and [4]. A closed-form ESPRIT-like method (see [4]) constructs an estimator based on the maximum overlapping two subarrays. However, it has been pointed out in [9] that any two subarrays configuration may be inherently suboptimal.

In this paper, we consider the spatial signature estimation problem with ULA in the presence of unknown gain and phase errors. The practicality of spatial signature can be found in [8] and [4]. Unlike ESPRIT-like method, our algorithm fully exploits the multiple invariances into ULA even though the array model errors exist. The sensor gain and phase errors are different from mutual coupling among elements of array and it can not be affected by other sensors. Furthermore, the steering matrix of ideal ULA has a Vandermonde structure. Both of them provide the possibility of selecting subarrays from uncalibrated array. It is natural to expect that an estimator formulated by multiple invariances in the array performs better than the one involved only one invariance. In practice, a multidimensional procedure of minimizing a cost function is presented. Our method firstly estimates the rotational DOA's (not the absolute DOA's) and error parameters, then obtains the estimation of spatial signature. The Gauss-Newton algorithm [10] is suggested for solving the multidimensional search problem. The choice of the initial values is essential for the global convergence of the iterative method. The excellent initial inputs are provided by ESPRIT-like method. Computer 
simulations show that only two or three iterations are required to achieve acceptable estimation values.

\section{DATA MODEL}

A uniform linear array with $M$ sensors received narrowband signals from $p$ far-field sources and the vector response $\boldsymbol{y} \in$ $\mathcal{C}^{M \times 1}$ of the array at time $t$ can be expressed as

$$
\boldsymbol{y}(t)=\boldsymbol{\Gamma}(\gamma) \boldsymbol{A}(\boldsymbol{\theta}) \boldsymbol{s}(t)+\boldsymbol{n}(t),
$$

where $s(t) \in \mathcal{C}^{p \times 1}$ is the vector of incident signals at time $t$, $\boldsymbol{n}(t) \in \mathcal{C}^{M \times 1}$ is the vector of additive noises, the ideal array steering matrix $\boldsymbol{A}(\boldsymbol{\theta})=\left[\begin{array}{llll}\boldsymbol{a}\left(\theta_{1}\right) & \boldsymbol{a}\left(\theta_{2}\right) & \cdots & \boldsymbol{a}\left(\theta_{p}\right)\end{array}\right]$, and

$$
\boldsymbol{a}(\theta)=\left[\begin{array}{llll}
1 & e^{j \frac{2 \pi}{\lambda} d \sin (\theta)} & \cdots & e^{j \frac{2 \pi}{\lambda}(M-1) d \sin (\theta)}
\end{array}\right]^{T} .
$$

Here, $\theta_{1}, \theta_{2}, \cdots, \theta_{p}$ are the Directions-of-Arrival of signals, $d$ and $\lambda$ represent the distance between two consecutive sensors and the identical wavelength for all signals, respectively. The matrix $\boldsymbol{\Gamma}(\boldsymbol{\gamma})=\operatorname{diag}\left[\begin{array}{cccc}\gamma_{1} & \gamma_{2} & \cdots & \gamma_{M}\end{array}\right]$ and $\left|\gamma_{i}\right|>0$ denotes the deterministic unknown gain and phase error of sensor $i$.

Two assumptions need to be made. Firstly, $s(t)$ is a temporally complex white Gaussian random vector with mean zero and its covariance matrix $\boldsymbol{R}_{\boldsymbol{s}}$ has full rank $p$ (assuming no correlate signals). Secondly, $\boldsymbol{n}(t)$ is a temporally and spatially complex white Gaussian random vector with mean zero and uncorrelated with incident signals.

The focus of this paper is the estimation of Spatial Signature matrix $\boldsymbol{V}=\boldsymbol{\Gamma}(\gamma) \boldsymbol{A}(\boldsymbol{\theta})$ from the $N$ snapshots of the array outputs. One ambiguity for this problem may be observed between the unknown signal vector $\boldsymbol{s}(t)$ and $\boldsymbol{V}$ (i.e., $\boldsymbol{V} \boldsymbol{s}(t)=\alpha \boldsymbol{V} \cdot\left(\frac{1}{\alpha} \boldsymbol{s}(t)\right)$ for an unknown non-zero scaling $\left.\alpha\right)$. A reasonable constraint for solving this scaling ambiguity is to let the first element of diagonal matrix $\Gamma(\gamma)$ be equal to one.

\section{ESTIMATION ALGORITHM}

A new subspace approach is presented for estimating spatial signature matrix $\boldsymbol{V}$ in this section. The so-called signal subspace $\boldsymbol{E}_{s}$ and noise subspace $\boldsymbol{E}_{n}$ can be obtained from the array outputs covariance matrix $\boldsymbol{R}=E\left\{\boldsymbol{y}(t) \boldsymbol{y}^{H}(t)\right\}$.

The Vandermonde structure of the ideal array steering matrix $\boldsymbol{A}(\boldsymbol{\theta})$ permits optimally exploiting multiple invariances for ULA even though the gain and phase of the sensor has not been calibrated. It is expected that an algorithm, fully making use of the particular properties, should obtain superior results than ESPRIT-like estimator proposed in [4]. Assume that the array comprises $n$ identical subarrays of $m$ sensors. Obviously, overlapping subarrays make $M \leq m n$. In addition, since the one-to-one correspondence between rows in $\boldsymbol{V}$ and elements of array, extracting a subarray from array is equivalent to picking up $m$ rows of matrix $\boldsymbol{V}$ by a $m \times M$ selection matrix $\boldsymbol{J}_{i}$. The full row rank matrix $\boldsymbol{J}_{i}$ consists of zeros and ones. Only one element at every row is equal to one and it corresponds to the selected sensor of the array.
The determinant of the matrix $\Gamma(\gamma)$ is given by

$$
\operatorname{det}(\boldsymbol{\Gamma}(\boldsymbol{\gamma}))=\prod_{i=1}^{M} \gamma_{i} \neq 0
$$

then the Rank of the matrix $\boldsymbol{V}$ is $p$, i.e., $\operatorname{Rank}(\boldsymbol{\Gamma}(\boldsymbol{\gamma}) \boldsymbol{A}(\boldsymbol{\theta}))=p$. It is clear that the matrix $\boldsymbol{\Gamma}(\gamma) \boldsymbol{A}(\boldsymbol{\theta})$ spans the same space as the signal subspace $\boldsymbol{E}_{s}$. The relation implies that there exists a nonsingular $p \times p$ matrix $\boldsymbol{T}$ satisfying

$$
\boldsymbol{J}_{i} \boldsymbol{E}_{s}=\boldsymbol{J}_{i} \boldsymbol{\Gamma}(\gamma) \boldsymbol{A}(\boldsymbol{\theta}) \boldsymbol{T}
$$

Considering $n$ subarrays, we have

$$
\boldsymbol{J}_{s}=\left[\begin{array}{c}
\boldsymbol{E}_{s 1} \\
\boldsymbol{E}_{s 2} \\
\boldsymbol{E}_{s 3} \\
\vdots \\
\boldsymbol{E}_{s n}
\end{array}\right]=\left[\begin{array}{l}
\boldsymbol{\Gamma}_{1} \boldsymbol{A}_{1} \\
\boldsymbol{\Gamma}_{2} \boldsymbol{A}_{1} \boldsymbol{\Phi}^{\varepsilon_{1}} \\
\boldsymbol{\Gamma}_{3} \boldsymbol{A}_{1} \boldsymbol{\Phi}^{\varepsilon_{2}} \\
\vdots \\
\boldsymbol{\Gamma}_{n} \boldsymbol{A}_{1} \boldsymbol{\Phi}^{\varepsilon_{n-1}}
\end{array}\right] \boldsymbol{T}
$$

where the matrix $\boldsymbol{J}$ is formed by selection matrix $\boldsymbol{J}_{i}$ and $\boldsymbol{J}=$ $\left[\begin{array}{llll}\boldsymbol{J}_{1}^{T} & \boldsymbol{J}_{2}^{T} & \cdots & \boldsymbol{J}_{n}^{T}\end{array}\right]^{T}, \boldsymbol{E}_{s i}, \boldsymbol{\Gamma}_{i}$ and $\boldsymbol{A}_{1}$ may be calculated by the following equations:

$$
\boldsymbol{E}_{s i}=\boldsymbol{J}_{i} \boldsymbol{E}_{s}, \quad \boldsymbol{\Gamma}_{i}=\boldsymbol{J}_{i} \boldsymbol{\Gamma}(\gamma) \boldsymbol{J}_{i}^{T}, \quad \boldsymbol{A}_{1}=\boldsymbol{J}_{1} \boldsymbol{A}(\boldsymbol{\theta}) .
$$

The matrix $\boldsymbol{\Phi}$ is given by

$$
\boldsymbol{\Phi}=\operatorname{diag}\left[e^{e^{\frac{2 \pi}{\lambda} d \sin \left(\theta_{1}\right)}} \quad \cdots \quad e^{j \frac{2 \pi}{\lambda} d \sin \left(\theta_{p}\right)}\right] .
$$

$\varepsilon_{i} d$ denotes the distance between the $(i+1)$ th subarray and the reference subarray. Analogous to MI ESPRIT method in [11], the unknown parameter vector $\boldsymbol{\mu}$ may now be obtained from the following least squares problem

$$
\Omega(\boldsymbol{\mu})=\left\|\left[\begin{array}{c}
\boldsymbol{E}_{s 1} \\
\boldsymbol{E}_{s 2} \\
\boldsymbol{E}_{s 3} \\
\vdots \\
\boldsymbol{E}_{s n}
\end{array}\right] \boldsymbol{W}^{1 / 2}-\left[\begin{array}{l}
\boldsymbol{\Gamma}_{1} \boldsymbol{A}_{1} \\
\boldsymbol{\Gamma}_{2} \boldsymbol{A}_{1} \boldsymbol{\Phi}^{\varepsilon_{1}} \\
\boldsymbol{\Gamma}_{3} \boldsymbol{A}_{1} \boldsymbol{\Phi}^{\varepsilon_{2}} \\
\vdots \\
\boldsymbol{\Gamma}_{n} \boldsymbol{A}_{1} \boldsymbol{\Phi}^{\varepsilon_{n-1}}
\end{array}\right] \boldsymbol{T}\right\|_{F}^{2}
$$

and

$$
\boldsymbol{\mu}=\left[\begin{array}{ccccccc}
\bar{\gamma}_{2} & \cdots & \bar{\gamma}_{M} & \tilde{\gamma}_{2} & \cdots & \tilde{\gamma}_{M} \\
\rho_{1} & \cdots & \rho_{p} & \theta_{1} & \cdots & \theta_{p}
\end{array}\right]^{T},
$$

where $\boldsymbol{W}$ is a Hermitian positive definite weighting matrix, $\bar{\gamma}_{i}$ and $\tilde{\gamma}_{i}$, respectively, denote the real part and image part of scaling $\gamma_{i}$. Since the signal subspace $\boldsymbol{E}_{s}$ is usually substituted with $\hat{\boldsymbol{E}}_{s}$ obtained from the eigendecomposition of the sample covariance matrix, the unitary matrix $\Phi$ in (7) becomes

$$
\boldsymbol{\Phi}=\operatorname{diag}\left[\begin{array}{lll}
\rho_{1} e^{j \frac{2 \pi}{\lambda} d \sin \left(\theta_{1}\right)} & \cdots & \rho_{p} e^{j \frac{2 \pi}{\lambda} d \sin \left(\theta_{p}\right)}
\end{array}\right] .
$$

Next, for ease of notation, we define

$$
\boldsymbol{\Upsilon}=\boldsymbol{W}^{T / 2}\left[\begin{array}{llll}
\boldsymbol{E}_{s 1}^{T} & \boldsymbol{E}_{s 2}^{T} & \cdots & \boldsymbol{E}_{s n}^{T}
\end{array}\right]
$$

and

$$
\boldsymbol{\Psi}=\left[\begin{array}{llll}
\boldsymbol{A}_{1}^{T} \boldsymbol{\Gamma}_{1} & \boldsymbol{\Phi}^{\varepsilon_{1}} \boldsymbol{A}_{1}^{T} \boldsymbol{\Gamma}_{2} & \cdots & \boldsymbol{\Phi}^{\varepsilon_{n-1}} \boldsymbol{A}_{1}^{T} \boldsymbol{\Gamma}_{n}
\end{array}\right] .
$$


The expression in (8) is reformulated as

$$
\Omega(\boldsymbol{\mu})=\left\|\mathbf{\Upsilon}-\boldsymbol{T}^{T} \boldsymbol{\Psi}\right\|_{F}^{2} .
$$

Here, the equations $\boldsymbol{\Phi}^{\varepsilon_{i} T}=\boldsymbol{\Phi}^{\varepsilon_{i}}$ and $\boldsymbol{\Gamma}_{i}^{T}=\boldsymbol{\Gamma}_{i}$ are used. This is a nonlinear least squares problem for unknown vector $\boldsymbol{\mu}$ (see, [10]). Solving $\boldsymbol{T}^{T}$ by minimizing the cost function $\Omega$, we can obtain

$$
\hat{\boldsymbol{T}}^{T}=\Upsilon \mathbf{\Psi} \boldsymbol{\Psi}^{H}\left(\boldsymbol{\Psi} \boldsymbol{\Psi}^{H}\right)^{-1}
$$

Substituting this back to (13) yields to

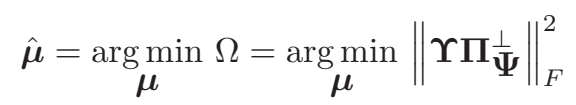

where

$$
\boldsymbol{\Pi}_{\mathbf{\Psi}}^{\perp}=\boldsymbol{I}-\boldsymbol{\Psi}^{H}\left(\boldsymbol{\Psi} \boldsymbol{\Psi}^{H}\right)^{-1} \boldsymbol{\Psi} .
$$

Note that, it is reasonable to involve all of the elements of the array for estimating unknown parameters. The limiting case is that there are no overlapping subarrays (i.e., $M=m n$ ). Thus, the matrix $\Psi$ has full row rank except for deleting some sensors from the array (i.e., $M<m n)$. So the matrix $\left(\Psi \Psi^{H}\right)$ is nonsingular.

\section{ALGORITHM IMPLEMENTATION}

The method considered herein requires a multidimensional search over parameter vector $\boldsymbol{\mu} \in \mathcal{R}^{2(M+p-1) \times 1}$. It is well known that the Gauss-Newton algorithm [10], [12] is a classical way to obtain the solution of this problem. Considering the cost function $\Omega$ in (15), the unknown parameters can be iteratively obtained by

$$
\boldsymbol{\mu}^{k+1}=\boldsymbol{\mu}^{k}-\xi_{k} \boldsymbol{H}^{-1} \boldsymbol{Q},
$$

where $\boldsymbol{\mu}^{k}$ denotes the estimate at $k$ iteration, $\xi_{k}$ is a iterative step length, the gradient vector $\boldsymbol{Q}=\frac{\partial \Omega}{\partial \boldsymbol{\mu}}$ and the Hessian matrix $\boldsymbol{H}=\frac{\partial^{2} \Omega}{\partial \boldsymbol{\mu} \partial \boldsymbol{\mu}^{T}}$ are evaluated at $\boldsymbol{\mu}^{k}$.

We define a vector $\boldsymbol{r}$ formed by columns scanning matrix $\Upsilon \Pi \frac{\perp}{\Psi}$, i.e.,

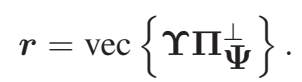

Then, the cost function $\Omega$ becomes

$$
\Omega=\boldsymbol{r}^{H} \boldsymbol{r} .
$$

In the following, based on the above equation, the expressions for the gradient vector and an approximate Hessian matrix are presented. Firstly, considering the gradient of $\Omega$ w.r.t $\boldsymbol{\mu}_{i}$, the $i$ th element of the gradient vector $\boldsymbol{Q}$ is given by

$$
\boldsymbol{Q}_{i}=\frac{\partial \Omega}{\partial \boldsymbol{\mu}_{i}}=2 \operatorname{Re}\left\{\boldsymbol{r}_{i}^{H} \boldsymbol{r}\right\},
$$

where $\boldsymbol{r}_{i}=\frac{\partial \boldsymbol{r}}{\partial \boldsymbol{\mu}_{i}}$. The $i j$ th component of the Hessian matrix may be expressed as (see [12], [13])

$$
\boldsymbol{H}_{i j}=\frac{\partial^{2} \Omega}{\partial \boldsymbol{\mu}_{i} \partial \boldsymbol{\mu}_{j}} \simeq 2 \operatorname{Re}\left\{\boldsymbol{r}_{i}^{H} \boldsymbol{r}_{j}\right\} \text {. }
$$

Reasons for this approximation (i.e., ignoring the second derivative of $r$ ) can be found in [11], [12] and it brings two benefits for the Gauss-Newton iterative algorithm. One is that the approximate Hessian matrix is positive semidefinite and it guarantees $-\boldsymbol{H}^{-1} \boldsymbol{Q}$ to be decent direction. The other is that we only need to calculate the first derivative of $r$ instead of the second one. Furthermore, a useful modification to illconditioned cases is to usually use $(\boldsymbol{H}+\zeta \boldsymbol{I})$ in lieu of $\boldsymbol{H}$, where $\zeta$ is a scaling.

Next, we focus on the first derivative of vector $r$ in (18). By the aid of the differentiation of the projection matrix [10], $\boldsymbol{r}_{i}$ may be expressed as

$$
\boldsymbol{r}_{i}=-\operatorname{vec}\left\{\boldsymbol{\Upsilon}\left(\boldsymbol{\Psi}^{\dagger} \boldsymbol{\Psi}_{i} \boldsymbol{\Pi}_{\overline{\boldsymbol{\Psi}}}^{\perp}+\left(\boldsymbol{\Psi}^{\dagger} \boldsymbol{\Psi}_{i} \boldsymbol{\Pi}_{\overline{\boldsymbol{\Psi}}}^{\perp}\right)^{H}\right)\right\}
$$

where $\boldsymbol{\Psi}^{\dagger}=\boldsymbol{\Psi}^{H}\left(\boldsymbol{\Psi} \boldsymbol{\Psi}^{H}\right)^{-1}$ and $\boldsymbol{\Psi}_{i}=\frac{\partial \boldsymbol{\Psi}}{\partial \boldsymbol{\mu}_{i}}$. The expression of $\boldsymbol{\Psi}_{i}$ can be obtained from (6), (9), (10) and (12). This is a tiresome calculation process. However, it can be simplified with the observation that $\frac{\partial \boldsymbol{\Phi}^{\varepsilon_{i-1}} \boldsymbol{A}_{1}^{T} \boldsymbol{\Gamma}_{i}}{\partial \gamma_{j}}=0$ if $\gamma_{j}$ can not be included in matrix $\boldsymbol{\Gamma}_{i}$, otherwise, $\frac{\partial \boldsymbol{\Phi}^{\varepsilon_{i-1}} \boldsymbol{A}_{1}^{T} \boldsymbol{\Gamma}_{i}}{\partial \bar{\gamma}_{j}}=\boldsymbol{\Phi}^{\varepsilon_{i-1}} \boldsymbol{A}_{1}^{T} \boldsymbol{I}_{k k}$ and $\frac{\partial \boldsymbol{\Phi}^{\varepsilon_{i-1}} \boldsymbol{A}_{1}^{T} \boldsymbol{\Gamma}_{i}}{\partial \tilde{\gamma}_{j}}=j \boldsymbol{\Phi}^{\varepsilon_{i-1}} \boldsymbol{A}_{1}^{T} \boldsymbol{I}_{k k}$, where $\boldsymbol{I}_{k k}$ is a matrix with one in the $k k$ th position and zeros elsewhere, $\gamma_{j}$ is the $k$ th element at the main diagonal of matrix $\Gamma_{j}$. Moreover,

$$
\frac{\partial \boldsymbol{\Phi}^{\varepsilon_{i-1}} \boldsymbol{A}_{1}^{T} \boldsymbol{\Gamma}_{i}}{\partial \theta_{j}}=\left(\frac{\partial \boldsymbol{\Phi}^{\varepsilon_{i-1}}}{\partial \theta_{j}} \boldsymbol{A}_{1}^{T}+\boldsymbol{\Phi}^{\varepsilon_{i-1}} \frac{\partial \boldsymbol{A}_{1}^{T}}{\partial \theta_{j}}\right) \boldsymbol{\Gamma}_{i},
$$

and

$$
\frac{\partial \boldsymbol{\Phi}^{\varepsilon_{i-1}} \boldsymbol{A}_{1}^{T} \boldsymbol{\Gamma}_{i}}{\partial \rho_{j}}=\frac{\partial \boldsymbol{\Phi}^{\varepsilon_{i-1}}}{\partial \rho_{j}} \boldsymbol{A}_{1}^{T} \boldsymbol{\Gamma}_{i}
$$

From the above analyses, the vector $\boldsymbol{r}_{i}$ can be calculated conveniently.

The proposed algorithm now is briefly outlined below.

1) Estimate the signal subspace $\hat{\boldsymbol{E}}_{s}$ from the eigendecomposition of the sample covariance matrix $\hat{\boldsymbol{R}}=$ $\frac{1}{N} \sum_{n=1}^{N} \boldsymbol{y}(n) \boldsymbol{y}^{H}(n)$, where $N$ is the finite number of snapshots.

2) Initialize vector $\boldsymbol{\mu}$ utilizing ESPRIT-like method [4].

3) Update $\boldsymbol{\mu}$ according to (17) until the stopping criterion is satisfied, then form the estimates of $\hat{\gamma}$ and $\hat{\boldsymbol{\theta}}$.

4) Calculate matrix $\boldsymbol{\Gamma}(\hat{\gamma})$ and $\boldsymbol{A}(\hat{\boldsymbol{\theta}})$, then spatial signature matrix is estimated as $\hat{\boldsymbol{V}}=\boldsymbol{\Gamma}(\hat{\gamma}) \boldsymbol{A}(\hat{\boldsymbol{\theta}})$.

Note that, although the ESPRIT-like method can only provide rotational values over true DOA's $\boldsymbol{\theta}$ and true gain and phase error matrix $\boldsymbol{\Gamma}$, it makes no difference to estimation of spatial signature $\boldsymbol{V}$. The identifiability of $\boldsymbol{V}$ from signal subspace $\boldsymbol{E}_{s}$ can be found in [4]. In addition, it is an intractable problem on how to choose weighting matrix $\boldsymbol{W}$. Refer to the weighted subspace fitting (WSF) method, we define

$$
\boldsymbol{W}_{\mathrm{opt}}=\left(\hat{\boldsymbol{\Lambda}}_{s}-\hat{\sigma}_{n}^{2} \boldsymbol{I}\right)^{2} \hat{\boldsymbol{\Lambda}}_{s}^{-1}
$$




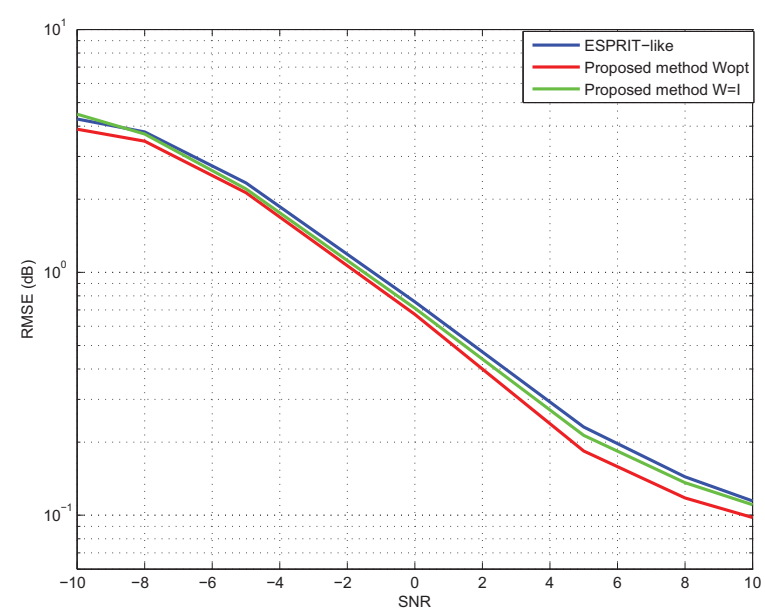

Fig. 1. RMSE of the spatial signature matrix $\boldsymbol{H}$ estimation versus SNR The number of snapshots $N=500$

where the diagonal matrix $\hat{\Lambda}_{s}$ contains the so-called signal eigenvalues estimated from the array outputs sample covariance matrix, $\hat{\sigma}_{n}^{2}$ denotes the consistent estimate of noise power. Simulation results using the proposed algorithm show that $\boldsymbol{W}=\boldsymbol{W}_{\text {opt }}$ results in a better performance for parameter estimates than $\boldsymbol{W}=\boldsymbol{I}$.

\section{EXPERIMENTS}

In this section, computer simulations were conducted for evaluating the performance of the proposed algorithm. In all scenarios, a ULA of 9 elements with half of wavelength element spacing is used. The ULA is divided into 5 subarrays and the $i$ th subarray is selected by matrix $\boldsymbol{J}_{i}=$ $\left[\mathbf{0}_{5 \times(i-1)}, \boldsymbol{I}_{5 \times 5}, \mathbf{0}_{5 \times(5-i)}\right], i=1, \cdots, 5$. The deterministic gain and phase errors are given by $1,1.10 e^{j 10^{\circ}}, 0.90 e^{-j 5^{\circ}}$, $1.25 e^{j 20^{\circ}}, 0.80 e^{-j 9^{\circ}}, 0.96 e^{j 15^{\circ}}, 1.18 e^{-j 23^{\circ}}, 0.88 e^{-j 2^{\circ}}$ and $0.85 e^{j 4^{\circ}}$. Two equal-power uncorrelated signals are considered and the SNR per element for each source is defined by $\mathrm{SNR}=10 \log _{10}\left(\sigma_{s}^{2} / \sigma_{n}^{2}\right)$, where $\sigma_{s}^{2}$ and $\sigma_{n}^{2}$, respectively, denote the power of incident signal and that of additive noise at each sensor. RMSE is used as the performance measure and defined by

$$
\operatorname{RMSE}=\frac{1}{K} \sum_{i=1}^{K} \sqrt{\left\|\hat{\boldsymbol{V}}_{i}-\boldsymbol{V}_{i}\right\|_{F}^{2}},
$$

where $K$ is the number of trials, $\hat{\boldsymbol{V}}_{i}$ and $\boldsymbol{V}_{i}$ are the estimated spatial signature and the true one at the $i$ th experiment, respectively. A total of 200 trials were performed for each simulation scenario.

\section{Example 1: Performance versus SNR}

In this example, two closed sources located at $25^{\circ}$ and $30^{\circ}$ were used. For comparison, we give the results of ESPRIT-like method [4] and the proposed method in this paper. Figure 1 shows that our method performs better than ESPRIT-like even in the weighting matrix $\boldsymbol{W}=\boldsymbol{I}$. It means that the maximum overlapping subarray is not the optimal choice in the presence

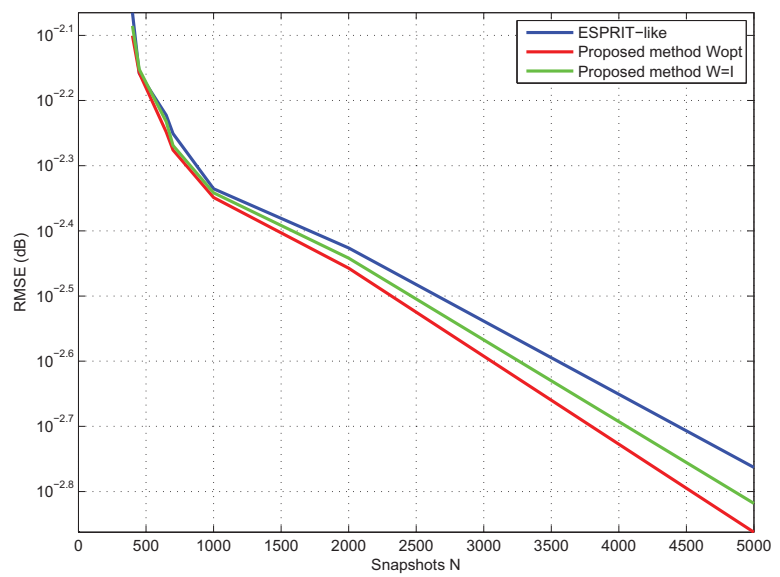

Fig. 2. RMSE of the spatial signature matrix $\boldsymbol{H}$ estimation versus the number of snapshots $N$. SNR $=15 \mathrm{~dB}$

of unknown sensor gain and phase responses. In other words, fully exploiting the multiple invariances in the array should be taken into account. In addition, adding a weighting matrix can evidently improve the performance of our algorithm.

Example 2: Performance versus Number of Snapshots

In the second case, the performance of different algorithms versus the number of snapshots $N$ were compared. The DOA's assumed to be $25^{\circ}$ and $40^{\circ}$. Similar conclusions to the Example 1 can be made from Figure 2. It is worth pointing out that the behavior of our method has been improved significantly at large number of snapshots. The reason may be that excellent initial input values are provided by the ESPRIT-like method.

Simulation results also show that our method only requires 2-4 Gauss-Newton iterations and the main performance improvement comes from the first two iterations.

\section{CONCLUSIONS}

A multidimensional search algorithm is presented for spatial signature estimation for ULA with unknown gain and phase errors. Our method fully excavates the multiple invariances of the ULA and is implemented with the help of the GaussNewton iterative algorithm. Because of excellent initial parameter values provided by ESPRIT-like, our method can converge rapidly to a appropriate solution. On the other hand, the introduction of a weighting matrix $W$ further improve the performance of the proposed algorithm. How to select subarrays from ULA, making the algorithm lead to optimal parameter estimation, is not addressed in this paper. Some pragmatic discussion about this question can be found in [9].

\section{ACKNOWLEDGMENT}

This work was supported in part by the National Nature Science Foundation of China under Grant 61172162.

\section{REFERENCES}

[1] R. Roy and T. Kailath, "Esprit-estimation of signal parameters via rotational invariance techniques," IEEE Trans. Acoust.,Speech, Signal Process., vol. 37, no. 7, pp. 984-995, 1989. 
[2] A. J. Weiss and B. Friedlander, "Eigenstructure methods for direction finding with sensor gain and phase uncertainties," Circuits Syst. Signal Process., vol. 9, no. 3, pp. 272-300, 1990.

[3] J. Pierre and M. Kaveh, "Experimental performance of calibration and direction-finding algorithms," in Proc. of IEEE ICASSP'91, Toronto, Ont., Apr. 1991, pp. 1365-1368.

[4] D. Astély, A. Swindlehurst, and B. Ottersten, "Spatial signature estimation for uniform linear arrays with unknown receive gains and phases," IEEE Trans. Signal Prosess., vol. 47, pp. 2128-2138, Aug. 1999.

[5] A. Paulraj and T. Kailath, "Direction of arrival estimation by eigenstructure methods with unknown sensor gain and phase," in Proc. of IEEE ICASSP'85, Tampa, FL, Apr. 1985, pp. 640-643.

[6] Y. Li and M. H. Er, "Theoretical analyses of gain and phase error calibration with optimal implementation for linear equispaced array," IEEE Trans. Signal Process., vol. 54, pp. 712-723, Feb. 2006.

[7] V. C. Soon, L. Tong, Y. F. Huang, and R. Liu, "A subspace method for estimating sensor gains and phases," IEEE Trans. Signal Process., vol. 42, pp. 973-976, Apr. 1994

[8] J. Gunther and A. Swindlehurst, "Algorithms for blind equalization with multiple antennas based on frequency domain subspaces," in Proc. of IEEE ICASSP'96, Atlanta, GA, May 1996, pp. 2419-2422.

[9] B. Ottersten, M. Viberg, and T. Kailath, "Performance analysis of the total least squares esprit algorithm," IEEE Trans. Signal Process., vol. 39, pp. 1122-1135, May 1991.

[10] G. H. Golub and V. Pereyra, "The differentiation of pseudo-inverses and nonlinear least squares problems whose variables separate," SIAM J. Numer. Anal., vol. 10, no. 2, pp. 413-432, 1973.

[11] A. Swindlehurst, B. Ottersten, R. Roy, and T. Kailath, "Multiple invariance esprit," IEEE Trans. Signal Prosess., vol. 40, pp. 867-881, Apr 1992.

[12] M. Viberg, B. Ottersten, and T. Kailath, "Detection and estimation in sensor arrays using weighted subspace fitting," IEEE Trans. Signal Process., vol. 39, pp. 2436-2449, Nov. 1991.

[13] M. Viberg and B. Ottersten, "Sensor array processing based on subspace fitting," IEEE Trans. Signal Process., vol. 39, pp. 1110-1121, May 1991. 